\title{
Pharyngitis and Pharyngeal Space Infections
}

Fever, Sore Throat, Difficulty Swallowing

Susannah Orzell and Amar Suryadevara

5.1 Introduction to the Problem - 54

5.2 Definitions -54

5.2.1 Cavities of the Head and Neck -54

5.2.2 Spaces of the Head and Neck - 54

5.2.3 Important Structures of the Head and Neck - 56

5.3 Basic Concepts -56

5.4 Infectious Causes of Pharyngitis and Parapharyngeal Infections - 56

5.4.1 Viruses as Causes of Pharyngitis and Related Infections - 57

5.4.2 Fungi as Causes of Pharyngitis and Related Infections - 58

5.4.3 Parasites as Causes of Pharyngitis and Related Infections - 58

5.4.4 Bacteria as Causes of Pharyngitis and Related Infections - 59

5.4.5 Deep Neck Space Infections - 60

5.5 Imaging for Deep Space Neck Infections - 63

5.6 Complications of Bacterial Pharyngitis and Deep Neck Space Infections - 64

5.7 Summary -64

$5.8 \quad$ Exercises -65

References -65 


\section{Learning Objectives}

- Understand that most cases of pharyngitis are caused by viruses and will resolve with supportive care alone.

- Describe common symptoms associated with a case of uncomplicated pharyngitis.

- Recognize the warning signs of a more serious infection requiring prompt attention such as a deep neck space infection, epiglottitis, or Ludwig's angina.

- Recognize some of the predisposing factors associated with atypical causes of pharyngeal infections.

\subsection{Introduction to the Problem}

Acute pharyngitis affects a significant number of people across the United States and the world each year and impacts patients from all age groups. It accounts for $1-2 \%$ of all ambulatory visits and causes missed days from work and school $[1,2]$. The cause is typically infectious in etiology, with viruses being most common pathogens involved, followed by bacteria, fungi, and rarely parasites. Noninfectious conditions may cause pharyngitis, and if symptoms are not self-limiting or do not respond to appropriate medical treatment, they should be considered. Symptoms range from a mild sore throat lasting for several days to severe manifestations that threaten the patency of the airway. Deep neck space infections can also extend directly into the mediastinum or the lungs causing life-threatening mediastinitis or pneumonia, underscoring the importance of early recognition and treatment.

\subsection{Definitions}

Important cavities, spaces, and structures in the head and neck. See Figs. 5.1, 5.2, and 5.3 for pictographic demonstrations of select neck spaces.

\subsubsection{Cavities of the Head and Neck}

Oral cavity - The anatomic cavity bound anteriorly by the lips, laterally by the buccal mucosa, inferiorly by the floor of the mouth, superiorly by the hard palate, and posteriorly by the anterior tonsillar pillar, circumvallate papillae, and junction of the hard and soft palates. Nasopharyngeal cavity - The anatomic cavity bound by the skull base superiorly, the soft palate inferiorly, the nasal choanae anteriorly, the arch of the atlas posteriorly, and the eustachian tubes laterally.

Oropharyngeal cavity - The anatomic cavity bound anteriorly by the anterior tonsillar pillar, the circumvallate papillae, and the junction of the hard and soft palates. The superior and middle constrictors form the posterior and lateral boundaries, and the soft palate forms the superior boundary. The tongue base and hyoid bone form the inferior boundary.

Hypopharyngeal cavity - The anatomic cavity extending from the level of the hyoid bone superiorly to the level of the cricoid inferiorly, the larynx anteriorly and cervical vertebrae 3 through 6 posteriorly, and the thyroid cartilage laterally.

\subsubsection{Spaces of the Head and Neck}

Peritonsillar space - This anatomic space is bound by the anterior tonsillar pillar anteriorly, the posterior tonsillar pillar posteriorly, the capsule of the palatine tonsil medially, and the superior pharyngeal constrictor laterally. The peritonsillar space is comprised of loose areolar connective tissue in most individuals, but the lingual branch of

- Fig. 5.1 Shown is a normal axial CT scan with intravenous contrast outlining the carotid sheath, parapharyngeal, retropharyngeal, parotid, and masticator spaces

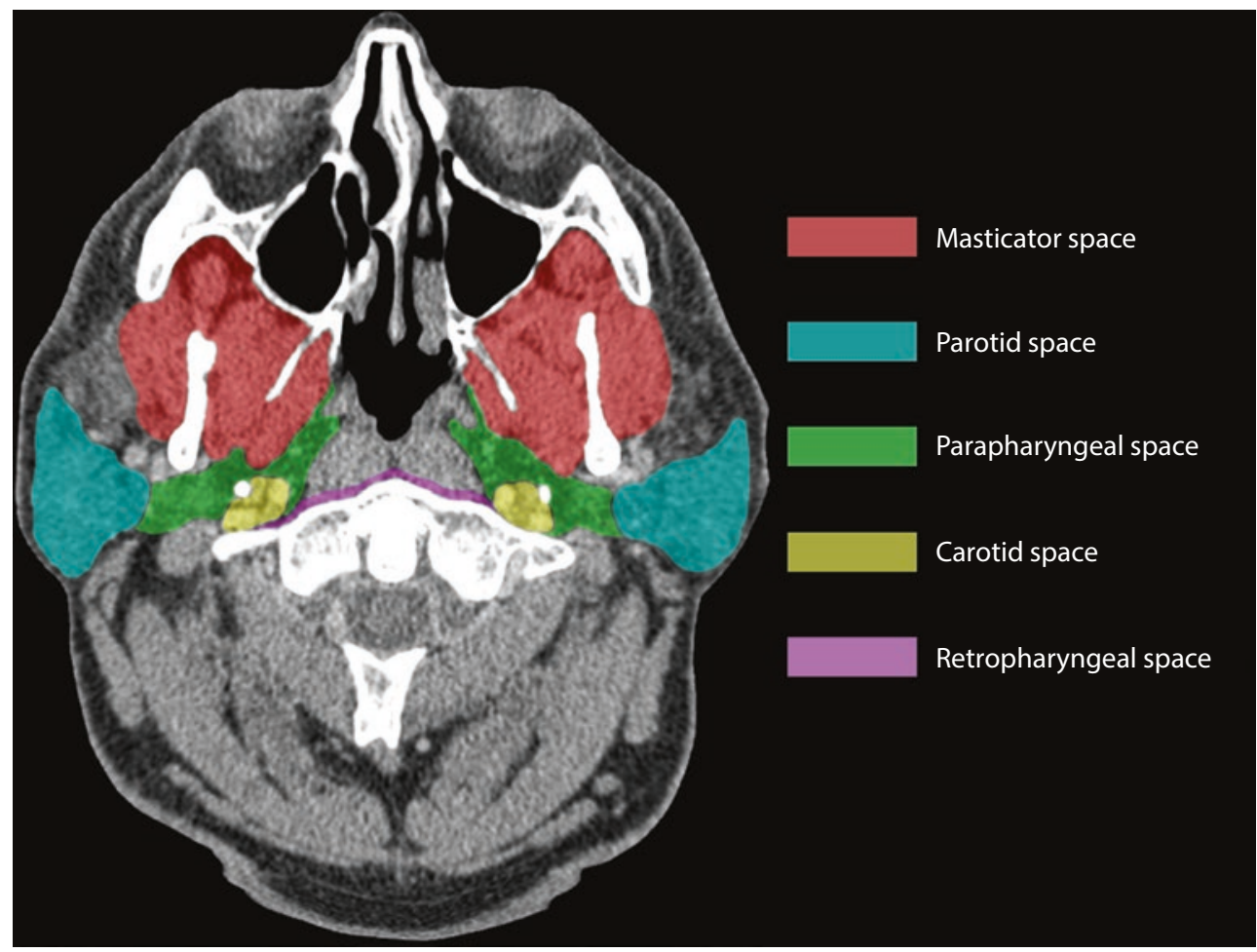


- Fig. 5.2 Shown is a normal axial CT scan with intravenous inferior position, now including the peritonsillar spaces contrast outlining the spaces outlined in Fig. 5.1 at a more

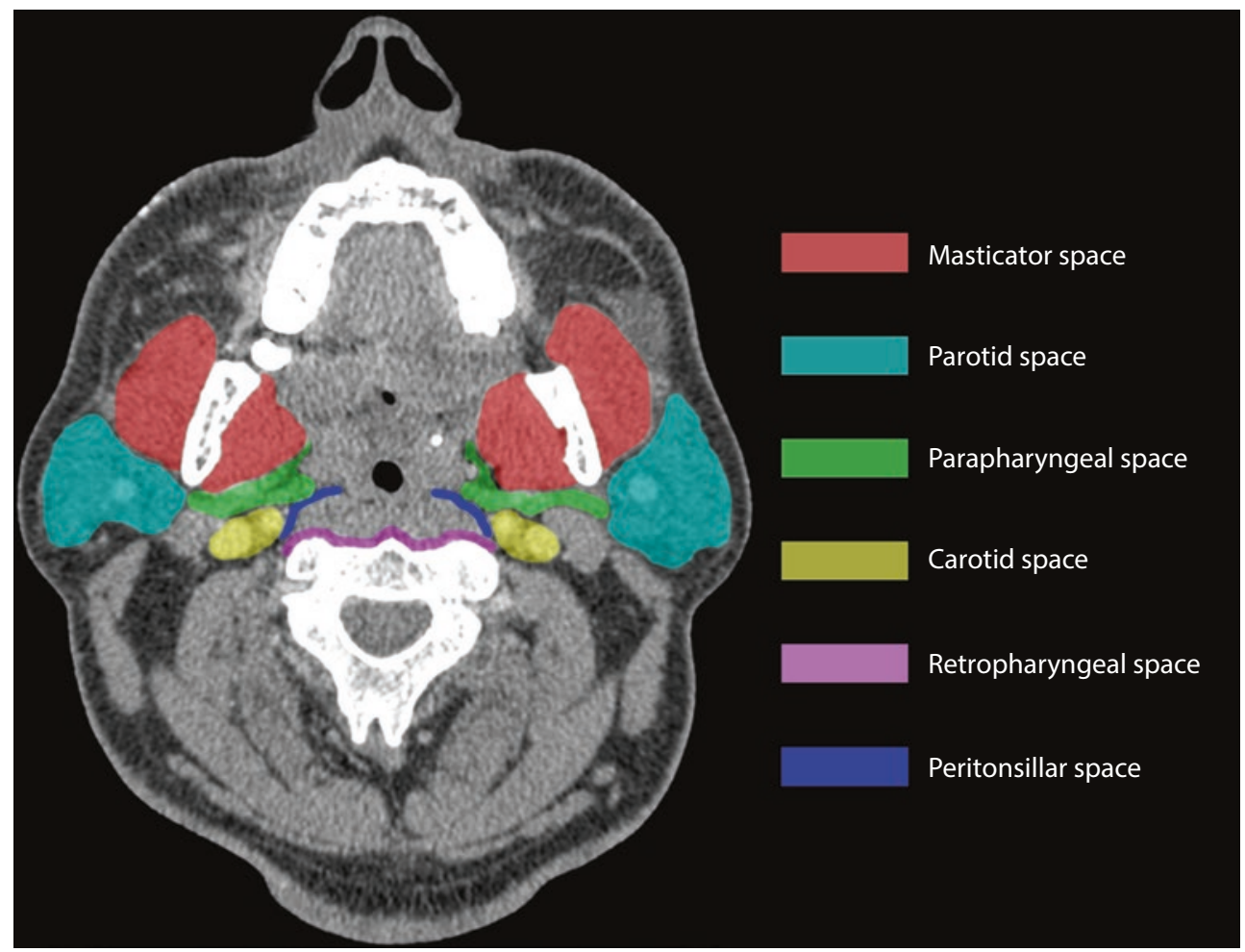

- Fig. 5.3 Shown is a normal axial CT scan with intravenous contrast outlining the carotid sheath, retropharyngeal, sublingual, and submandibular spaces

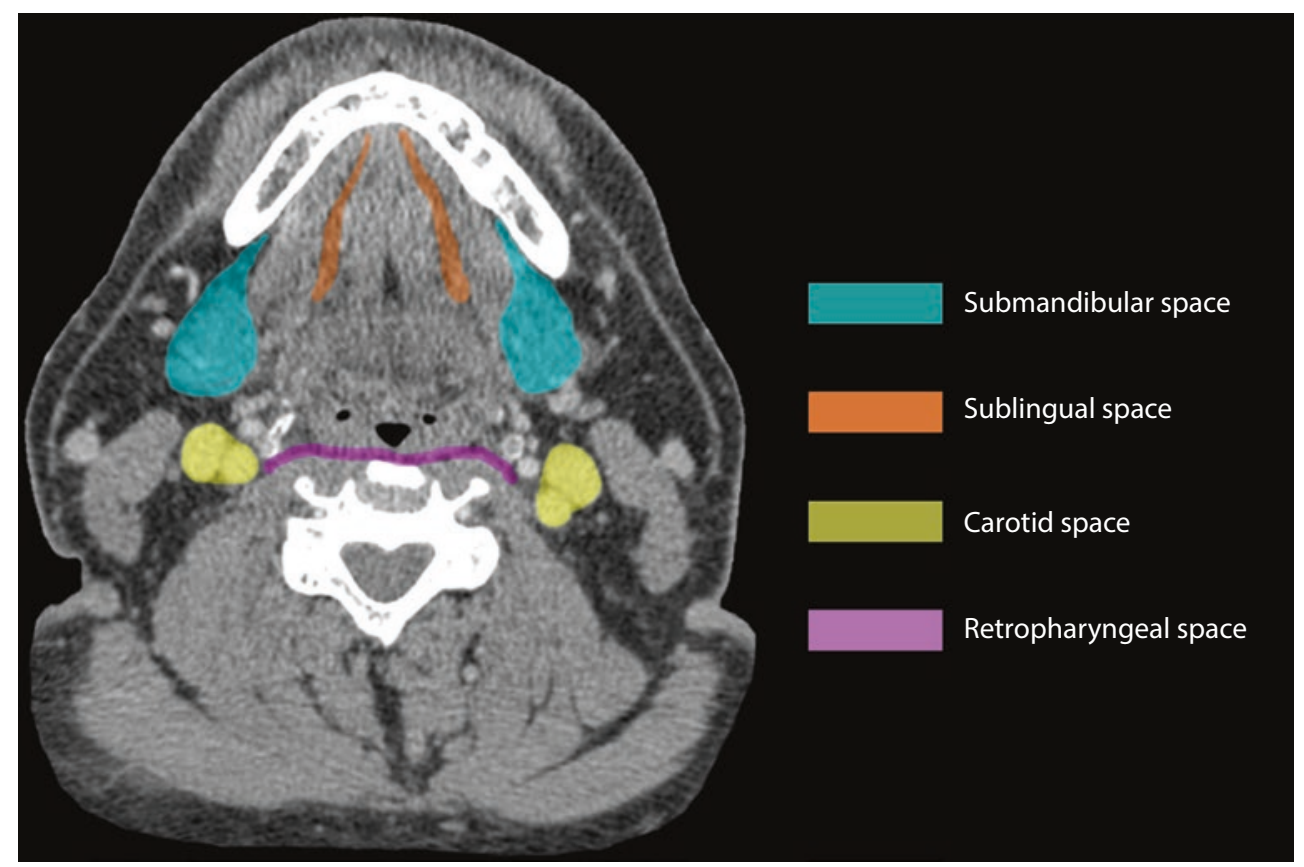

the glossopharyngeal nerve courses through it in a minority as an anatomic variant. The peritonsillar space communicates with the parapharyngeal space.

Parapharyngeal space - This anatomic space is traditionally described as an inverted pyramid lateral to the peritonsillar space and nasopharyngeal cavity. It is bound superiorly by the base of the skull, inferiorly by the greater cornu of the hyoid bone, anteriorly by the pterygomandibular raphe and medial pterygoid, posteriorly by the cervical vertebrae and paravertebral muscles, and laterally by the parotid gland. The parapharyngeal space is further divided into preand post-styloid compartments by a band of fascia called the aponeurosis of Zuckerkandl and Testut, which connects the styloid process of the temporal bone to the tensor veli palatini. The pre-styloid compartment contains fat, muscles, lymph nodes, the deep lobe of the parotid gland, the internal maxillary branch of the external carotid artery, and several branches of cranial nerves. The post-styloid compartment contains the internal carotid artery, internal jugular vein, sympathetic chain, lymph nodes, and cranial nerves IX through XII. This space communicates with the submandibular space, peritonsillar space, retropharyngeal space, and sublingual space.

Retropharyngeal space - As its name implies, this anatomic space lies posterior to the pharynx. It is bound anteriorly by the visceral 
division of the middle cervical fascia, posteriorly by the alar division of the deep cervical fascia, laterally by the carotid sheaths, superiorly by the skull base, and inferiorly to the mediastinum. This space typically only contains loose areolar connective tissue and lymph nodes, but may contain the internal carotid arteries as an anatomic variant referred to as retropharyngeal carotids. Infections in this space are particularly concerning due to their ability to spread inferiorly to the mediastinum or superiorly to the skull base. The retropharyngeal space communicates with the parapharyngeal space.

Danger space - This anatomic space lies just posterior to the retropharyngeal space, and like the retropharyngeal space, it extends from the skull base to the mediastinum. It is bound posteriorly by the prevertebral fascia and laterally by the transverse processes of the vertebrae. It is called the "danger space" because bacterial infections in this area can spread to the thorax very quickly.

Prevertebral space - This anatomic space lies posterior to the prevertebral fascia and danger space and extends from the skull base to the coccyx. It is bound posteriorly by the vertebral bodies and laterally by the transverse processes of the vertebrae. It contains muscles, the vertebral arteries and veins, and the phrenic nerve and roots of the brachial plexus.

Submandibular space - This anatomic space is bound anteriorly by the anterior belly of the digastric muscle, posteriorly by the posterior belly of the digastric muscle, medially and superiorly by the mylohyoid muscle, laterally by the platysma, and inferiorly by the hyoid bone. It contains the submandibular gland, lymph nodes, facial vessels, and hypoglossal nerve. It communicates with the parapharyngeal and sublingual spaces.

Sublingual space - These anatomic paired spaces are each bound superiorly by the floor of the mouth, inferiorly by the mylohyoid muscle, anteriorly and laterally by the mandible, posteriorly by the hyoid bone, and medially by extrinsic muscles of the tongue. They communicate with each other beneath the lingual frenulum, and they communicate with the submandibular and parapharyngeal spaces.

Masticator space - This anatomic space is bound medially by the fascia medial to the pterygoid muscles, laterally by the mandibular ramus, superiorly by the base of the skull, inferiorly by the lower border of the mandible, anteriorly by the pterygomandibular raphe, and posteriorly by the parotid gland. It is subdivided into the masseteric and pterygoid spaces, which are delineated by the superficial layer of the deep cervical fascia. The masticator space contains muscles of mastication, the ramus and posterior body of the mandible, the inferior alveolar nerve, and the internal maxillary branch of the external carotid artery. This space communicates with the parapharyngeal space, submandibular space, and sublingual spaces.

\subsubsection{Important Structures of the Head and Neck}

Waldeyer's tonsillar ring - An arrangement of lymphoid tissue in the pharyngeal cavity that forms a circular loop consisting of the lingual tonsils, the adenoids, and the palatine tonsils.

Carotid sheath - Paired anatomic structures lateral to the retropharyngeal space extending from the base of the skull to the sternum containing the carotid arteries, internal jugular veins, cranial nerves IX through XII, and the sympathetic trunk.

\subsection{Basic Concepts}

The vast majority of patients with pharyngitis have mild to moderate illness that is either self-limiting or rapidly responsive to appropriate antibiotic treatment. The initial approach
Call Out Box 5.1

Deep neck space infections can cause airway compromise. It is important to recognize signs of impending respiratory failure and establish a safe airway before any other interventions are started.

to a patient with suspected pharyngitis or parapharyngeal space infection, however, should always include a careful assessment for signs of airway compromise. Findings of concern include respiratory distress, drooling, orthopnea, muffled speech ("hot potato voice"), difficulty turning the head, and elevation of the tongue and floor of the mouth. A patient in acute distress due to a retropharyngeal abscess, for example, may exhibit opisthotonic posturing with neck hyperextension. These findings should prompt immediate transfer to an emergency department for evaluation with flexible laryngoscopy, if expertise is available. In extreme cases, emergency endotracheal intubation or surgical interventions are necessary to establish and protect the airway. Intravenous broadspectrum antibiotics should also be administered as soon as possible in critical patients. The laboratory evaluation typically includes a complete blood count, erythrocyte sedimentation rate, C-reactive protein, blood cultures (both aerobic and anaerobic), and pharyngeal, laryngeal, tracheal, or epiglottis cultures for those undergoing a procedure that protects their airway. Imaging should be deferred for the critically ill patient until their airway has been secured. The recognition of and immediate intervention for a compromised airway are always the top priority (airway, breathing, circulation, in that order).

In the stable patient, the extent of the diagnostic evaluation depends on the history and physical examination findings. The history should include a determination of the onset, progression and duration of symptoms, the quality and severity of any associated pain, and factors that aggravate or alleviate the discomfort. The presence or absence of associated symptoms, such as fever, cough, dysphagia, trismus, nasal congestion, rhinorrhea, neck pain, or dental pain, should be noted. Symptoms that are more prominent on one side suggest the presence of an abscess or phlegmon. The presence or absence of underlying chronic medical conditions, particularly those that are associated with immunosuppression such as diabetes, HIV infection, collagen vascular diseases, or malignancy, should be determined. A recent history of dental work, endotracheal intubation, surgery on the upper aerodigestive tract, and/or penetrating neck trauma is also important to obtain [ Call Out Box 5.1].

\subsection{Infectious Causes of Pharyngitis and Parapharyngeal Infections}

Infectious etiologies of pharyngitis and parapharyngeal infections include viruses, bacteria, fungi, and parasites. By far, viral infections are the most common. Susceptibility to 
Table 5.1 Typical causes of viral pharyngitis

bidities, and exposures, so a complete history is necessary to guide clinical decision-making or to raise the possibility that a more unusual pathogen may be responsible for a patient's illness. Rarely, autoimmune disorders, previously unrecognized immunodeficiency, anatomic abnormalities, or malignancies can exacerbate or mimic symptoms of neck infections. Patients with persistent symptoms, who do not respond to empiric therapy based on the suspected diagnosis of infection, should undergo additional diagnostic testing to evaluate for the presence of the more unusual infectious and noninfectious entities.

\subsubsection{Viruses as Causes of Pharyngitis and Related Infections}

Most cases of pharyngitis and tonsillopharyngitis are caused by viruses [3]. Since effective antiviral medications are not available for the majority of relevant viruses, treatment is nearly always focused on reducing pain and fever. Patients may refuse to eat and drink for a sufficient period of time, making intravenous fluids necessary to reestablish and maintain hydration. - Table 5.1 lists the more common causes of viral pharyngitis. Pharyngitis is usually one of several symptoms that patients complain about when infected with any of the common "cold and flu" viruses. Since respiratory viral pathogens do not discriminate between mucosal targets, symptoms of conjunctivitis, rhinitis, and laryngitis with cough and/or hoarseness are typically present along with the sore throat. If only a single mucous membrane appears to be involved, a bacterial cause should be considered.

Any of the respiratory viral infections can be associated with fever, especially in young children, but influenza viruses and adenoviruses are notorious for causing fevers at any age. Viral pharyngitis is a clinical diagnosis. Respiratory virus diagnostic testing is available, however, for patients with severe infection or confusing atypical presentations. At the present, polymerase chain reaction (PCR)-based testing can be performed on nasopharyngeal or oropharyngeal samples. Some platforms allow for the rapid diagnosis of more than a dozen viruses simultaneously.

Respiratory "cold and flu" viruses account for most cases of viral pharyngitis, but several others are also worthy of discussion. For example, exudative pharyngitis is a classic finding in patients with infectious mononucleosis, a syndrome most commonly caused by Epstein-Barr virus (EBV). The syndrome is diagnosed clinically in patients with exudative pharyngitis, lymphadenopathy, and splenomegaly. Fevers are common and can persist for 3 weeks or longer. Patients with infectious mononucleosis also complain of extreme fatigue, sometimes lasting for months. The diagnosis of EBV-associated infectious mononucleosis is confirmed serologically by testing for $\operatorname{IgM}$ and IgG antibodies against the EBV viral capsid antigen (EBV VCA IgM and IgG). The detection of EBV VCA IgM is consistent with acute EBV infection. A rapid screening test for EBV infection, the serum heterophil antibody assay, is still used

\begin{tabular}{|c|c|c|}
\hline Viruses & $\begin{array}{l}\text { Are they typical } \\
\text { "cold and flu" } \\
\text { viruses? }\end{array}$ & $\begin{array}{l}\text { Is effective antiviral } \\
\text { treatment available? }\end{array}$ \\
\hline Rhinoviruses & Yes & No \\
\hline Adenoviruses & Yes & No \\
\hline $\begin{array}{l}\text { Coronaviruses } \\
229 \mathrm{E}, \mathrm{HKU} 1, \mathrm{OC} 43 \text {, } \\
\text { NL63 }\end{array}$ & Yes & No \\
\hline $\begin{array}{l}\text { Respiratory } \\
\text { syncytial virus }\end{array}$ & Yes & No \\
\hline $\begin{array}{l}\text { Parainfluenza } \\
\text { viruses } 1 \text { through } 4\end{array}$ & Yes & No \\
\hline $\begin{array}{l}\text { Influenza A and B } \\
\text { viruses }\end{array}$ & Yes & Yes \\
\hline $\begin{array}{l}\text { Human } \\
\text { metapneumovirus }\end{array}$ & Yes & No \\
\hline Echoviruses & Yes & No \\
\hline $\begin{array}{l}\text { Coxsackie } A \text { and } B \\
\text { viruses }\end{array}$ & Yes & No \\
\hline Enteroviruses & Yes & No \\
\hline Epstein-Barr virus & No & No \\
\hline Cytomegalovirus & No & $\begin{array}{l}\text { Yes, but not used for } \\
\text { pharyngitis }\end{array}$ \\
\hline $\begin{array}{l}\text { Human } \\
\text { immunodeficiency } \\
\text { virus (HIV) }\end{array}$ & No & $Y_{e s}{ }^{a}$ \\
\hline $\begin{array}{l}\text { Herpes simplex } \\
\text { viruses } 1 \text { and } 2\end{array}$ & No & $\begin{array}{l}\text { Yes, but seldom used } \\
\text { for pharyngitis }\end{array}$ \\
\hline
\end{tabular}

${ }^{a}$ Antiretroviral medications are not typically used specifically for the symptoms of pharyngitis associated with the acute retroviral syndrome of newly acquired HIV infection

commonly. Results should be interpreted with caution since the assay is both less sensitive and less specific than antibody testing. Treatment for EBV infection is primarily supportive care. In severe cases, the combination of pronounced pharyngeal swelling and impressive cervical adenopathy may lead to concerns for impending airway compromise. Under such circumstance, systemic glucocorticoids can be administered to reduce the swelling, perhaps avoiding the need for a medical or surgical procedure to maintain airway patency. The use of glucocorticoids should otherwise be avoided because of their immunosuppressive activity.

The second most common cause of infectious mononucleosis is cytomegalovirus. The clinical presentation can be identical to that seen from EBV, but the EBV-specific serologic testing will not indicate an acute EBV infection, and the rapid heterophil antibody assay will be negative. A positive CMV IgM antibody test confirms the diagnosis of acute CMV infection. 
Acute infection with human immunodeficiency virus (HIV) can also present with an infectious mononucleosislike illness called acute retroviral syndrome. The syndrome is characterized by self-limiting fevers, malaise, myalgias, pharyngitis, and cervical lymphadenopathy occurring several days to several weeks after exposure. HIV should, therefore, be suspected in all patients who present with an infectious mononucleosis syndrome. The diagnosis of HIV infection is made using combined, fourth-generation tests designed to detect both HIV-specific antibodies and HIV p24 antigen. Patients who are infected recently, who have not yet seroconverted by making anti-HIV antibodies, often have detectable circulating HIV p24 antigen. A major advantage of fourth-generation testing is the ability to detect HIV p24 antigen and diagnose HIV infection as early as 1 week after exposure. The earliest detectable anti-HIV antibody is present after 2-4 weeks.

Herpes simplex virus (HSV) types 1 and 2 can also cause impressive pharyngitis. Patients may present with fevers, malaise, headaches, cervical lymphadenopathy, and sore throat with or without the formation of vesicles visible in the oropharyngeal cavity. When vesicles are seen, they tend to rupture forming ulcerative plaques with grayish exudate.

Infants and young children who develop primary oral HSV infection typically present with gingivostomatitis. A very painful vesicular eruption is seen on the mucous membranes in the anterior part of the mouth including the gingiva and the tongue, with lesions extending onto the lips. In contrast, primary oral HSV infection in adolescents and adults most commonly presents as severe pharyngitis. Since vesicles are not always present, it is likely that a substantial number of such cases go undiagnosed. The diagnosis of HSV pharyngitis (or gingivostomatitis) is made either by HSV-specific polymerase chain reaction (PCR)-based testing or by viral culture of material collected by swabbing the affected mucous membranes. In immunocompetent patients, oral HSV infections will eventually resolve spontaneously; however, outpatient treatment of antiviral medication acyclovir, or one of its derivatives, offers the potential to reduce symptoms, reduce virus shedding, and hasten recovery. Immunosuppressed patients who develop active HSV disease benefit most by treatment with intravenous acyclovir.

Another group of viruses capable of causing painful vesicular lesions in the mouth are the enteroviruses. The term herpangina is used to describe these lesions when present on the roof of the mouth and in the back of the throat. Despite its name and the vesicular nature of the lesions, herpangina refers to an enteroviral infection, not a herpetic infection. When herpangina is associated with a maculopapular or vesicular rash of the hands and feet, it is referred to as hand, foot, and mouth disease. Among the enteroviruses that cause herpangina with or without the rash, coxsackie A16 is the most notorious because it continues to be responsible for a large number of pediatric cases of hand, foot, and mouth disease each summer during enterovirus season. The diagnoses of herpangina and hand, foot, and mouth disease are easy to make on clinical grounds alone, but if viral diagnostic testing is pursued, PCR testing is pre- ferred. Enterovirus typing is only performed by reference laboratories and usually only for the purposes of outbreak investigations.

\subsubsection{Fungi as Causes of Pharyngitis and Related Infections}

Oral candidiasis, commonly called "thrush," is the most common fungal infection of the upper aerodigestive tract. Thrush leads to white, cheese-like plaques on the tongue and buccal mucosa that are not easily scraped away. Some bleeding may occur if scrapings are done for diagnostic purposes. The diagnosis of oral candidiasis is made clinically; however if scrapings of the affected area are cultured, the yeast Candida albicans or a related Candida species is recovered. Two less typical presentations of oral candidiasis include the erythematous and the chronic hyperplastic types. Erythematous oral candidiasis presents with a red, very sore oropharyngeal cavity, while chronic hyperplastic candidiasis presents with leukoplakia at the corners of the mouth and tongue. C. albicans is a normal flora of the human skin and mucous membranes. When there is a disturbance in immune function or an imbalance in the usual bacteria flora, as occurs with antibiotic use, C. albicans can cause infection. Thrush is quite common during the first few months of life, at least in part because of the immaturity of the newborn's cellular immune function. In almost every other instance, $C$. albicans requires a conditional opportunity to cause an opportunistic infection. Patients who are being treated with long-term antibiotics or immunosuppressive medications, including glucocorticoids, and those with primary and acquired immune deficiencies commonly develop thrush. Untreated, the infection can progress and extend to posterior pharyngeal cavity structures, the esophagus, and the airway. Pharyngitis secondary to candidiasis can be extremely painful causing severe dysphagia. When oral candidiasis progresses to visibly involve the structures of the posterior oropharyngeal cavity, it is important to consider that it may have also spread to the esophagus, larynx, or trachea. Direct visualization using nasopharyngeal laryngoscopy may be indicated. The presence of advanced oral candidiasis in a patient without known risk factors should always prompt an evaluation for immunocompromising conditions, including HIV infection.

Other fungal infections of the pharynx and parapharyngeal space caused by a variety of opportunistic yeasts and molds have been described as case reports, nearly always in patients with significant immunocompromising conditions.

\subsubsection{Parasites as Causes of Pharyngitis and Related Infections}

Parasitic infections of the pharynx and parapharyngeal spaces are exceedingly rare but should be considered in certain circumstances. In developed countries, infection with Toxoplasma gondii has been described in organ transplanta- 
tion patients being treated with immunosuppressive medications and in patients with poorly controlled HIV infection. Case reports of pharyngeal space infections caused by tapeworms (Echinococcus granulosus and Taenia solium), roundworms (Lagochilascaris minor), and protozoa (Leishmania braziliensis and T. gondii) have all been reported to occur. The diagnosis of a parasitic infection of the pharyngeal cavity or parapharyngeal space requires a high index of suspicion, surgical sampling, and expert parasitologists working in the clinical microbiology laboratory. Treatment would depend on the parasite involved, usually in collaboration with experts at the US Centers for Disease Control and Prevention and/or World Health Organization.

\subsubsection{Bacteria as Causes of Pharyngitis and Related Infections}

Bacterial infections are second to viral infections as the most common group of pathogens to cause pharyngitis. Streptococcus pyogenes, which is also referred to as group A streptococcus, is the most common bacterial cause of pharyngitis. Bacteria also cause nearly all deep suppurative infections of the head and neck. The deep space infections are often polymicrobial. Culture results are representative of the diverse microbiologic flora of the normal human oropharynx [4-9] ( $>$ Box 5.1), although virulent pathogens such as Staphylococcus aureus and $S$. pyogenes are also identified on a regular basis. The etiologies and sequelae of these infections have been shown which vary by patient characteristics, particularly by age and by the presence of comorbid conditions. For example, dental infections are the most common source and predisposing factor for deep neck space infections in adults, but tonsillitis and pharyngitis are the most common predisposing factor among children [10-12]. Adolescents and young adults have higher rates of peritonsillar abscess compared to the younger children or older adults, while retropharyngeal abscesses are most common in preschool-aged children [13].

\subsubsection{Bacterial Causes of Pharyngitis and Tonsillopharyngitis}

Group A streptococcus (GAS) is the most common cause of acute bacterial pharyngitis and tonsillopharyngitis. Less frequent causes are listed in - Table 5.2. Clinically, streptococcal pharyngitis is associated with fever, tender anterior cervical lymphadenopathy, pharyngeal erythema, and tonsillar swelling. Exudate may be seen on the posterior pharyngeal wall and on the tonsils. Streptococcal pharyngitis may also be associated with the presence of palatal petechiae and a skin rash. The diagnosis of GAS pharyngitis is based on both the clinical presentation and the results of laboratory testing. The Centor and modified Centor criteria
Box 5.1 Bacterial Causes of Deep Neck Space

Infections

Virulent invasive pathogens that can also

be oropharyngeal flora

- Staphylococcus aureus

- Streptococcus pyogenes (group A beta-hemolytic streptococcus)

- Haemophilus influenzae

Oropharyngeal flora: facultative anaerobes

- Viridans group streptococcia

- Other Streptococcus species

- Haemophilus parainfluenzae

- Moraxella catarrhalis

- Eikenella corrodens

- Cutibacterium acnes

- Mycobacterium species other than tuberculosis

Oropharyngeal flora: obligate anaerobes

- Fusobacterium necrophorum

- Bacteroides fragilis group ${ }^{\mathrm{b}}$

- Prevotella species

- Porphyromonas species

aThe viridans group streptococci are alpha-hemolytic Streptococcus species, many of which are normal species of the human gastrointestinal tract. There are six major groups: $S$. mutans, S. salivarius, S. anginosus, S. mitis, S. sanguinis, and S. bovis ${ }^{\mathrm{b}}$ The Bacteroides fragilis group includes $B$. fragilis (the most common), Bacteroides distasonis, Bacteroides ovatus, Bacteroides thetaiotaomicron, and Bacteroides vulgatus
Table 5.2 Bacterial causes of pharyngitis

\begin{tabular}{l|l} 
Bacteria & Relative frequency \\
$\begin{array}{l}\text { Streptococcus pyogenes (group A } \\
\text { streptococcus) }\end{array}$ & Most common \\
$\begin{array}{l}\text { Streptococcus zooepidemicus and others } \\
\text { (group C streptococcus) }\end{array}$ & Common \\
\hline $\begin{array}{l}\text { Streptococcus dysgalactiae and others } \\
\text { (group G streptococcus) }\end{array}$ & Common \\
\hline Mycoplasma pneumoniae & Common \\
\hline $\begin{array}{l}\text { Arcanobacterium haemolyticum } \\
\text { Neisseria gonorrhoeae }\end{array}$ & Regular \\
\hline Fusobacterium necrophorum & Regular \\
\hline Corynebacterium diphtheriae & Regular \\
\hline Chlamydophila pneumoniae & Rare \\
\hline Chlamydia trachomatis & Rare \\
\hline Yersinia pestis & Rare \\
\hline Francisella tularensis & Rare \\
\hline Chlamydophila psittaci & Rare (pharyngeal \\
tularemia)
\end{tabular}

${ }^{a}$ Chlamydia trachomatis can be detected regularly from pharyngeal swabs, but is only rarely associated with symptoms of pharyngitis 
- Table 5.3 Modified Centor criteria for the clinical diagnosis of streptococcal pharyngitis

\begin{tabular}{|l|c|}
\hline Criteria & Points \\
\hline Absence of cough & 1 \\
\hline Swollen, tender anterior cervical lymph nodes & 1 \\
\hline Temperature $>100.4^{\circ} \mathrm{F}\left(38^{\circ} \mathrm{C}\right)$ & 1 \\
\hline Tonsillar exudates or swelling & 1 \\
\hline Age & \\
\hline $3-14$ years & 1 \\
\hline $15-44$ years & 0 \\
\hline $45+$ years & -1 \\
\hline
\end{tabular}

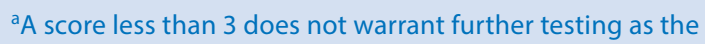
probability of a Group A streptococcal infection is low. A score of 3 or more should warrant further testing with cultures or rapid antigen detection tests and antibiotic treatment if these tests are positive

\section{Call Out Box 5.2}

Empiric treatment of pharyngitis with antibiotics is no longer recommended. Initiation of antibiotics should be directed by cultures.

(- Table 5.3) have been used as diagnostic adjuncts to help clinicians identify patients who are likely to have GAS infection and unlikely to have a viral infection. A score of 0 or 1 on the modified Centor score indicates a low probability of GAS infection, and no further diagnostic testing or empiric antibiotic therapy is recommended. Score of 2 or 3 indicates the possibility of strep throat, so throat cultures are recommended, with initiation of antibiotics if the cultures are positive for group A streptococcus. A score of 4 or more once indicated that empiric treatment with antibiotics should be started while waiting for culture results; however, in recent years, the US Centers for Disease Control and Prevention (CDCP) and national professional societies have since recommended against empiric antibiotic usage [ $>$ Call Out Box 5.2]. Antibiotics should only be used when cultures are positive $[14,15]$. Overall, evidence has shown only a modest benefit of antibiotics in reducing the duration of a sore throat symptoms; however treatment is highly effective at preventing acute rheumatic fever and reduces the frequency of peritonsillar abscess formation [16]. Antibiotic treatment does not, however, reduce the possibility of developing post-streptococcal glomerulonephritis. Penicillin is the antibiotic of choice for GAS pharyngitis unless the patient is allergic to it. Amoxicillin is an acceptable alternative. Patients who are allergic to, or cannot tolerate, $\beta$-lactam antibiotics can be treated with clindamycin, azithromycin, or clarithromycin. Unlike many other common bacterial pathogens, $S$. pyogenes has not developed resistance to $\beta$-lactam antibiotics. Resistance to clindamycin is rare in most communities but well described in others. Occasional resistance to azithromycin and clarithromycin is also seen. Clinical treatment failures with penicillin and amoxicillin do occur despite the absence of antibiotic resistance. Such failures are best explained by failure of the penicillin pharmacodynamics, not by a resistance mechanism acquired by the streptococcus.

Some patients develop recurrent GAS pharyngitis and/or tonsillitis because unlike many other common infections, natural disease does not confer protective immunity to reinfection. The American Academy of Otolaryngology clinical practice guidelines for streptococcal pharyngitis advocate for watchful waiting if a patient has had fewer than seven documented episodes of streptococcal pharyngitis in the last year, fewer than five episodes per year for at least 2 years, or fewer than three infections per year for at least 3 years $[17,18]$. For patients who do not meet these criteria, tonsillectomy may be offered. In addition, tonsillectomy may be considered in patients with comorbidities, including obstructive sleep apnea, chronic tonsillitis unresponsive to medical therapy, cardiac valvular disease, recurrent febrile seizures, tonsiliths, history of peritonsillar abscess, or allergy/intolerance to antibiotic therapy. Ideally, a tonsillectomy should be performed in the absence of an active infection to reduce the chances of a surgical complication such as postoperative bleeding. Occasionally an emergency tonsillectomy, historically referred to as a quinsy tonsillectomy, needs to be performed because of airway compromise.

\subsubsection{Deep Neck Space Infections}

Deep neck space infections can present as discrete organized abscesses within specific neck spaces, as a soft tissue phlegmons without clearly forming collections of pus, or rarely, as a very rapidly destructive life-threatening process called necrotizing fasciitis. These conditions typically arise from the direct spread of a less serious infectious process present in an adjacent space. Deep neck space infections become particularly concerning when compartments that communicate directly with the mediastinum are involved, such as the retropharyngeal, danger, and prevertebral spaces. Depending on their specific location, these infections can also cause significant airway compromise over a relatively short period of time. The underlying predisposing cause for deep neck space infections varies based on age. Children who develop deep neck infections usually do so after starting with pharyngitis, while the most common initial source of infection in adults comes from an odontogenic process [ $>$ Call Out Box 5.3]. The

Call Out Box 5.3

Pharyngitis is the most common source for developing deep neck space infections in children, whereas odontogenic infections are the most common underlying source in adults. 


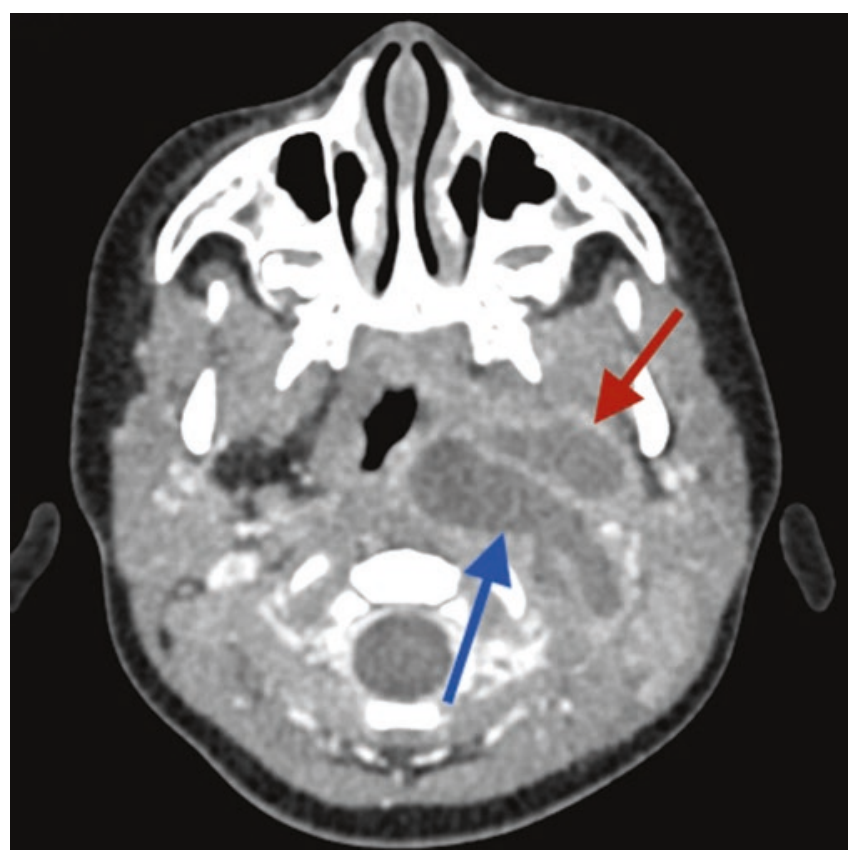

- Fig. 5.4 Shown is an axial CT scan demonstrating contiguous right-sided parapharyngeal (red arrow) and retropharyngeal abscesses (blue arrow)

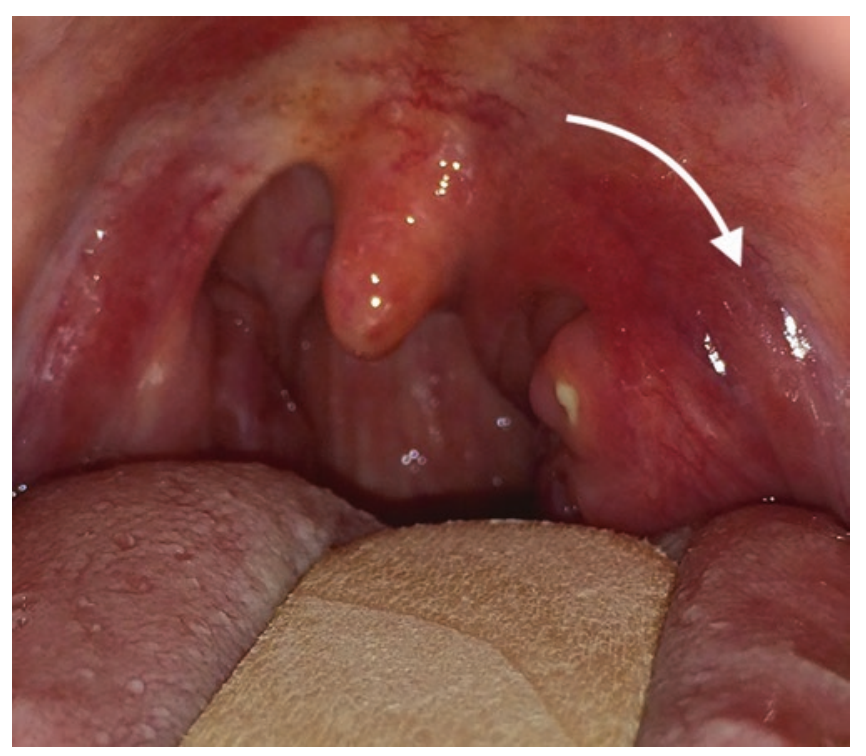

- Fig. 5.5 Cellulitis of the left anterior tonsillar pillar and soft palate in a patient with a small peritonsillar abscess (arrow)

Trismus is less frequent and, when present, less severe. Posterior parapharyngeal space abscesses are also associated with a higher incidence of sepsis due to their proximity to the carotid sheath.

Patients with RPAs often present in extremis, appearing toxic with severe respiratory distress, holding their neck in hyperextension and drooling. RPAs also cause bulging of the posterior pharyngeal wall (• Fig. 5.7).

Despite the numerous clinical findings that can use to diagnose and potentially differentiate between deep neck space infections, most cases will require imaging. The imagabscesses result in posterior pharyngeal wall swelling. 


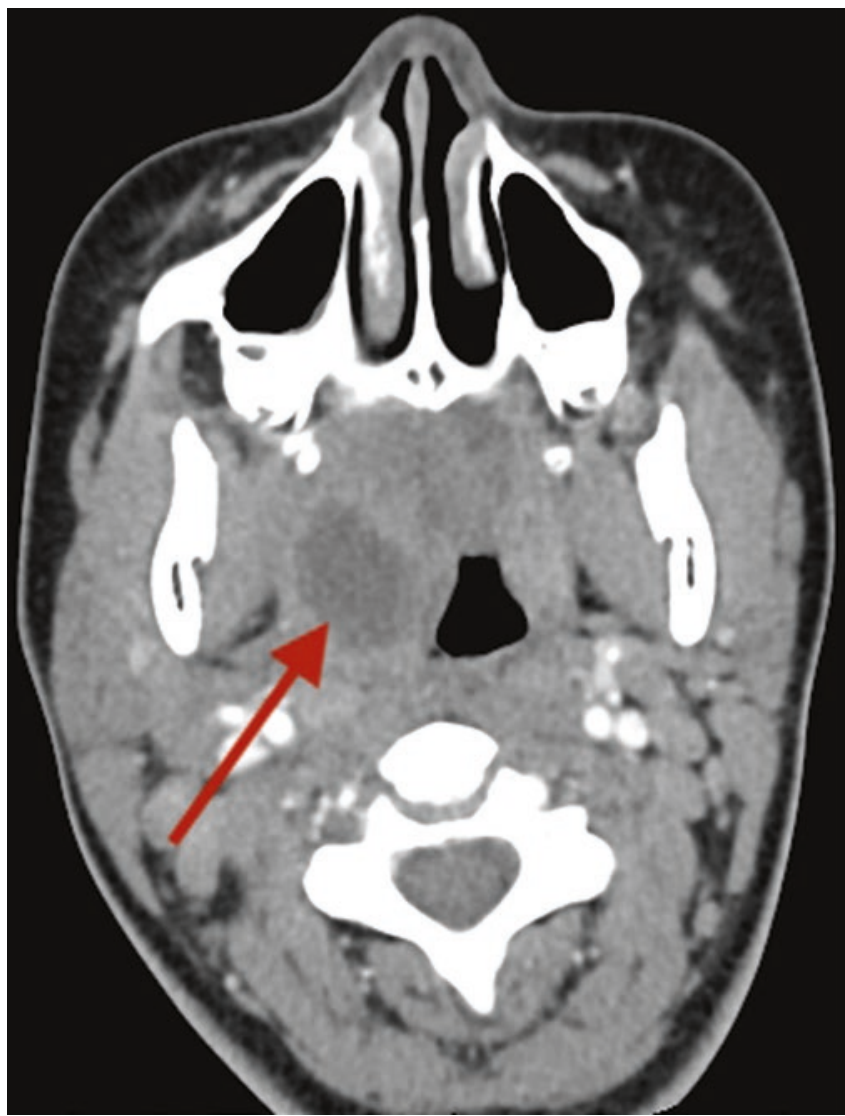

- Fig. 5.6 Shown is an axial CT scan establishing the presence of a left-sided peritonsillar abscess (arrow)

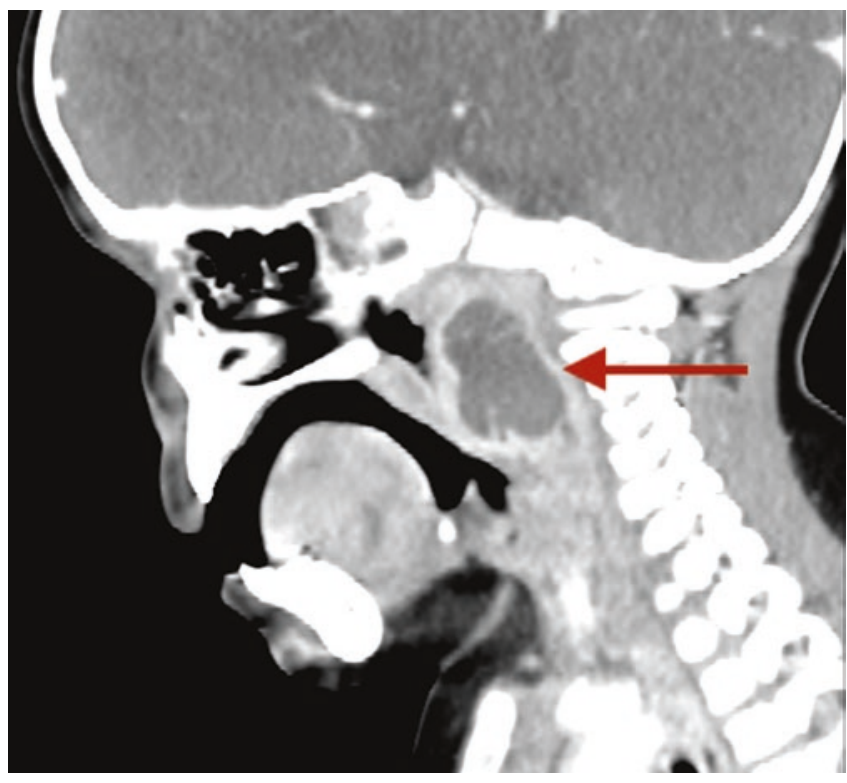

- Fig. 5.7 Shown is a sagittal CT scan showing a retropharyngeal abscess (arrow)

ing modality of choice is a contrast-enhanced CT scan of the neck. Some patients will not be able to tolerate lying supine and may require endotracheal intubation prior to the imaging procedure.
Call Out Box 5.4

Small, clinically mild peritonsillar abscesses, retropharyngeal abscesses, and parapharyngeal abscesses can be managed medically with close follow-up, whereas larger, more symptomatic abscesses require a surgical drainage procedure.

Needle aspiration or incision and drainage is a common method of treating abscesses in the head and neck, although intratonsillar abscesses are usually not treated surgically unless the tonsils become obstructive. Peritonsillar abscesses may be treated in a conscious patient using either needle aspiration or incision and drainage. General anesthesia is necessary for surgical drainage of other deep neck space abscesses. New evidence suggests that small PTAs, particularly those that are not associated with severe symptoms, can be treated medically, avoiding an incision and drainage procedure altogether [23]. Similarly, some small, clinically mild, parapharyngeal space abscesses $[20,24]$ and RPAs $[25,26]$ can be treated medically with close follow-up in case a surgical procedure becomes necessary due to progression of the illness [ $\vee$ Call Out Box 5.4].

Although suppurative infections of the deep neck spaces are often regarded as being more acute and in need of surgical drainage, nonsuppurative infections can be just as severe and, in some cases, more serious than deep neck space abscesses. One of these conditions is Ludwig's angina. This condition refers to a rapidly spreading cellulitic infection of the submental, sublingual, and submandibular areas, typically due to an infection originating in the teeth. The swelling of the sublingual space displaces the tongue superiorly and posteriorly. The swelling impairs speech and ability to swallow. As the infection progresses, the patency of the airway becomes compromised. On physical examination, the patient has a muffled voice and appears distressed. Trismus may prevent examination of the oropharynx. The structures of the superior neck are indurated with a marked elevation of the tongue and floor of mouth. Ludwig's angina is a surgical emergency. The treatment priority is establishing a protected airway, typically by tracheostomy. Once the airway is protected, the infection is incised and drained and the area debrided as necessary. Broad-spectrum intravenous antibiotics are used. Ideally the dental source of the infection is also addressed, but severe trismus typically prevents access during the incision and drainage procedure.

Cervical necrotizing fasciitis (CNF) is an uncommon, life-threatening infection of the head and neck associated with substantial morbidity and mortality. These infections typically arise from an existing infection of the adjacent deep neck spaces that propagates along the relatively avascular fascial planes. Because of the poor vascularity of the fascia, these infections are poorly responsive to antibiotic therapy and demand surgical debridement. Clinically and radiographically, CNF is difficult to differentiate from cellulitis or phlegmon. Symptoms typical of other pharyngeal 
infections, such as sore throat, dysphagia, and odynophagia, are present; however CNF may also cause bullae, skin necrosis, subcutaneous emphysema, and pain out of proportion to the findings on examination. Secondary obstruction of lymphatic drainage can give the skin an "orange-peel" appearance $[27,28]$.

The classic CT scan finding of gas bubble formation within the soft tissues of the neck (• Fig. 5.8) is found in 65\% of CNF cases [29]. Other CT scan findings typically include the presence of diffuse edematous changes of the surrounding soft tissue and scattered areas of hypodensity that do not enhance in the presence of contrast material. Laboratory-based predictors of necrotizing fasciitis, such as the laboratory risk indicator for necrotizing fasciitis (LRINEC) score [24] and the model developed by Wall et al. [30], which are validated from other anatomic areas of the body, have not proven useful in diagnosing CNF [29]. When clinical and radiographic findings suggest the possibility that a patient has CNF, surgical exploration is necessary. If the surgical team encounters necrotic tissue that is easily pulled apart, the diagnosis of $\mathrm{CNF}$ is confirmed, and debridement is performed. Tissue is debrided until there is a bleeding, viable edge. Repeat visits to the operating room for additional surgeries are expected.

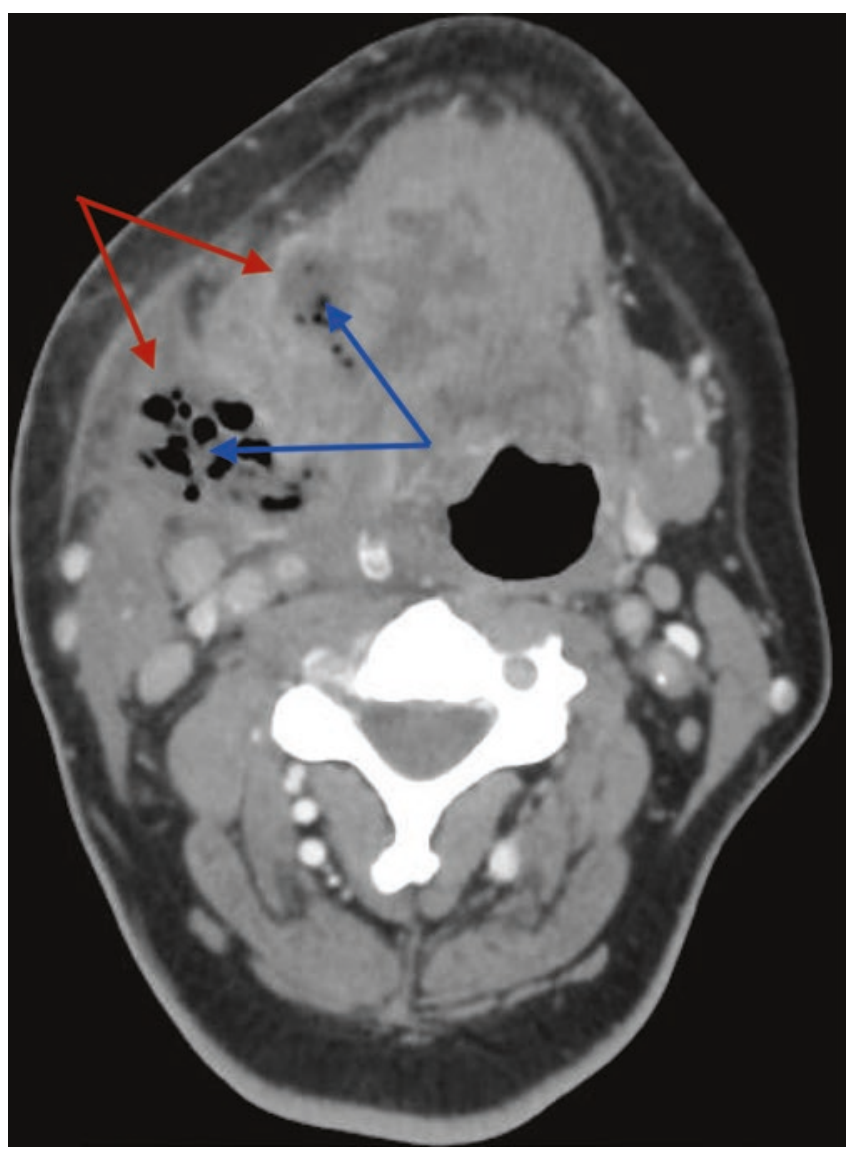

- Fig. 5.8 Shown is an axial CT scan of the neck revealing hypodense areas without peripheral enhancement (red arrows) and the abnormal presence of gas pockets (blue arrows) within the soft tissue. Surgical debridement of this area revealed necrotic tissue, confirming a diagnosis of necrotizing fasciitis
Medical treatment involves meticulous intensive care support and the administration of long-term, broad-spectrum intravenous antibiotics. Even with prompt recognition and treatment, CNF has a mortality rate approaching 40\% [31].

\subsection{Imaging for Deep Space Neck Infections}

The radiographic study of choice for the evaluation of a deep neck space infection is a contrast-enhanced CT scan. A CT scan provides excellent characterization of the soft tissues and bony structures. This is particularly important when there is a suspicion of an odontogenic source of infection (- Fig. 5.9). Abscesses appear as areas of hypodensity with rim enhancement. Other imaging modalities, including plain radiography, ultrasonography, and magnetic resonance imaging (MRI), may be considered. Plain radiographs and ultrasonography do not provide the resolution detail that a CT scan does, but may be useful in some instances. Panoramic radiographs used to evaluate dentition may also be used to identify odontogenic sources of infection. Ultrasonography allows for the possibility of concurrent evaluation and treatment of an abscess with drainage under guidance, although the inability of ultrasound to visualize deeper structures of the neck often limits its use. MRI is rarely used in the evaluation of acute head and neck infections, but may be considered in cases where extension to the skull base, intracranial compartment, prevertebral space, or other complications, such as thrombosis of critical vessels, are suspected.

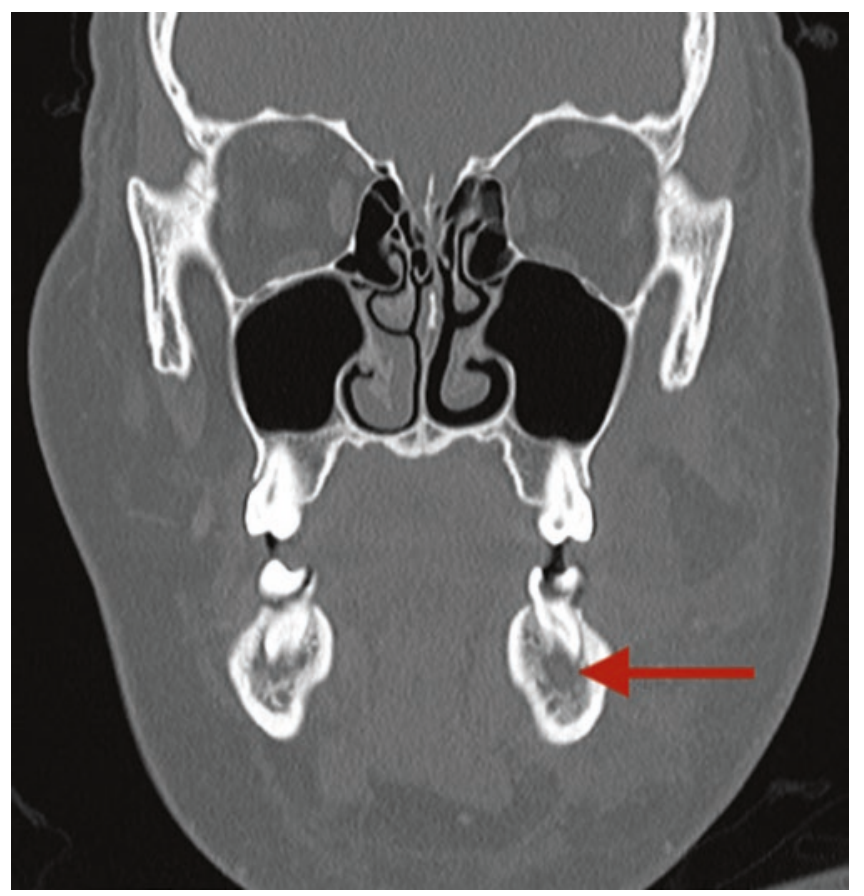

- Fig. 5.9 Shown is a coronal CT scan demonstrating a periapical lucency (arrow) in a patient with a submandibular abscess, indicating the dental source of the infection 


\subsection{Complications of Bacterial Pharyngitis and Deep Neck Space Infections}

Complications of bacterial pharyngitis and deep neck space infections are uncommon, particularly if appropriate antibiotic therapy has been instituted and immediate surgical concerns have been addressed; however direct extension and invasion into surrounding structures or spaces are always a risk. Other complications include bacteremia, Lemierre's syndrome, carotid artery aneurysm/rupture, and spread along adjacent neck spaces or along fascial planes.

Lemierre's syndrome occurs when infection, usually with the anaerobic bacterium Fusobacterium necrophorum, extends from the pharynx or parapharyngeal space to the internal jugular vein causing an infectious thrombophlebitis. This can lead to bacteremia as portions of the infected clot break away causing septic emboli. The lungs are typically affected first. Patients with septic metastasis of infection to the lungs may develop pulmonary nodules, cavities, and abscesses. Patients may also develop septic arthritis, particularly of the knee, hip, or shoulder [32]. The diagnosis of Lemierre's syndrome is confirmed by performing a contrastenhanced CT scan of the neck to demonstrate the presence of a thrombosed internal jugular vein. Treatment consists of intravenous antibiotics that include reliable activity against anaerobic bacteria. Some experts also recommend anticoagulation therapy. In rare instances, ligation or excision of the internal jugular vein may be necessary if the patient fails to respond to standard treatment [ $\vee$ Call Out Box 5.5].

Other potential complications of pharyngeal and parapharyngeal infections involving the carotid sheath are carotid artery aneurysm or rupture. These complications are extremely rare and only described in case reports. When they occur, they are life-threatening and demand immediate attention. Similar to Lemierre's syndrome, carotid artery pseudoaneurysm or rupture secondary to infection occurs due to spread of the infection from the pharynx or parapharyngeal spaces into the carotid sheath. Unlike Lemierre's syndrome, this condition tends to affect nonvascular structures in the carotid sheath, resulting in Horner syndrome and palsies of cranial nerves IX through XII. Damage to the walls of the carotid artery may produce a pulsatile neck mass on palpation, and in the case of carotid artery rupture, an expanding neck hematoma may be accompanied by a very large volume of bright red blood coming from the oropharynx. Treatment involves protecting the patient's airway and either surgical ligation or embolization to prevent circulatory collapse from blood loss.

Call Out Box 5.5

Lemierre's syndrome refers to infectious thrombophlebitis of the internal jugular vein that occurs as a complication of pharyngitis. It is most commonly caused by the obligate anaerobe, Fusobacterium necrophorum. Direct extension of the infection from the infected veins to the lungs causes a serious anaerobic bacterial pneumonia.
Spread of infection to the retropharyngeal space can lead to retropharyngeal abscesses as well as spread to other important areas of the body, such as the prevertebral space, danger space, or the mediastinum. The retropharyngeal, danger, and prevertebral spaces are bound by the skull base superiorly, and the retropharyngeal and danger spaces extend to the mediastinum, whereas the prevertebral space extends to the coccyx. Once infection reaches these spaces, there is relatively easy access to the mediastinum. The complication of mediastinal infection is rare, estimated to occur at a rate of 4.1/100,000 [33], but is always life-threatening $[34,35]$.

Complications specific to GAS pharyngitis include two noninfectious conditions: acute rheumatic fever (ARF) and post-streptococcal glomerulonephritis (PSGN). These entities are believed to arise from autoimmune complications secondary to the host's immune response to the GAS infection. The autoimmune sequelae seen with ARF are believed to be due to molecular mimicry, while PSGN occurs secondary to a type 3 hypersensitivity reaction.

ARF is rare within the developed world due to the availability of antibiotics; however it is still a significant cause of morbidity and mortality in developing countries $[36,37]$. Untreated or multiple episodes of ARF may progress to chronic rheumatic heart disease causing lifelong complications. Acutely, patients with ARF may present with fever, arthritis, arthralgia, carditis, first-degree heart block, erythema marginatum, Sydenham chorea, or subcutaneous nodules. Inflammatory biomarkers are typically elevated. A diagnosis of ARF is made by a combination of clinical and laboratory findings as outlined by the modified Jones criteria [36].

PSGN typically occurs several weeks after GAS infection. Unlike ARF, PSGN may occur despite effective treatment of the GAS infection. Symptoms of PSGN include hematuria, edema, and hypertension although many cases present with asymptomatic microscopic hematuria alone. Acute renal failure may need to be supported temporarily with dialysis, but PSGN typically resolves with time. A small number of patients go on to develop chronic renal failure.

\subsection{Summary}

Pharyngeal infections are very common, and not often lifethreatening. They are usually caused by viruses and resolve without the need for any intervention. Sepsis, muffled voice, difficulty breathing, drooling, inability to turn the head, and unilateral throat swelling on exam are not normal and demand immediate attention. Additionally, findings suggestive of an abscess on imaging warrant further evaluation and possibly surgical drainage. Failure to treat these patients in a timely manner may lead to further extension of the abscess, either within the same neck space or spread to a different neck or chest space, sepsis, invasion into surrounding 
vasculature, bacteremia, Lemierre's syndrome, or even death. It is also important to recognize that immunocompromised patients are at risk for different pathogens compared to immunocompetent patients.

\subsection{Exercises}

Please refer to the supplementary information section for answers to these exercises.

1. A 21-year-old male patient presents to your office for evaluation of a sore throat. He states that his sore throat began 3 days ago and is associated with a runny nose, cough, and a hoarse voice. He is afebrile and nontoxic in appearance and does not have tender cervical lymphadenopathy, but does have tonsillar exudates. He has had two episodes of GAS pharyngitis when he was a child. What is the most appropriate next step in treatment?
a. Manage conservatively
b. Begin empiric treatment with antibiotics
c. Perform a throat culture and begin empiric treatment with antibiotics
d. Perform a throat culture and use results to guide antibiotic treatment

2. A 2-year-old male patient presents to the ED in respiratory distress. His mother states that he has complained of throat pain for several days and that his voice has sounded muffled. He also developed fevers to $39^{\circ} \mathrm{C}$, and his oral intake has decreased to the point where he has not eaten for the past days. On physical examination, he is tachypneic, has a toxic appearance, and appears tired. He is sitting forward with his neck in a position of hyperextension, and he is drooling. What is your next step in the management of this patient?

a. Obtain an emergency CT scan of the neck

b. Obtain blood, urine, and cerebrospinal fluid cultures; initiate antibiotic treatment

c. Perform an airway evaluation, and establish a secure airway if needed

d. Perform a bedside ultrasound to assess for a drainable abscess

3. A 35-year-old female presents for evaluation of a sore throat. She has had the sore throat for approximately 2 weeks, and it has been worsening during this time. Additionally, she notes that her mouth hurts. She denies voice changes and difficulty breathing and states that her pain is not any worse on one side or the other. She denies any significant past medical history or use of medications, but does reveal that she is an intravenous drug user and occasionally will share needles. On physical examination, you find a diffuse white deposit over her oral mucosa that bleeds slightly when scraped. A presumptive diagnosis of oral candidiasis (thrush) is made. In addition to starting the patient on antifungal treatment, what other tests or treatments would be prudent in this patient?
a. Antibiotics
b. Fungal culture
c. HIV testing
d. None

4. A 7-year-old female presents to the emergency department for evaluation of a sore throat and difficulty eating. For the past 5 days, she has had worsening right-sided throat pain associated with fevers, chills, and a slight muffled voice. In the ED, she is febrile to $38.8^{\circ} \mathrm{C}$, but not in any respiratory distress. She is able to swallow without significant difficulty. During your physical examination, you note some fullness and erythema of the right anterior tonsillar pillar. A CT scan shows a small collection of hypodense fluid with rim enhancement in the right peritonsillar space. Acceptable options for the management of this patient include (more than one answer may apply):

a. Hospitalize for observation and treatment with intravenous antibiotics

b. Discharge home on oral antibiotics with follow-up in $24 \mathrm{~h}$

c. Perform a needle aspiration now

d. Take the patient to the operating room for an incision and drainage under general anesthesia

\section{References}

1. Schappert SM, Rechtsteiner EA. Ambulatory medical care utilization estimates for. Natl Health Stat Rep. 2006;2008(8):1-29.

2. Pfoh E, Wessels MR, Goldmann D, Lee GM. Burden and economic cost of group A streptococcal pharyngitis. Pediatrics. 2008;121(2): 229-34.

3. Bisno AL. Acute pharyngitis. N Engl J Med. 2001;344(3):205-11.

4. Ridder GJ, Technau-Ihling K, Sander A, Boedeker CC. Spectrum and management of deep neck space infections: an 8-year experience of 234 cases. Otolaryngol Head Neck Surg. 2005;133(5):709-14.

5. Flynn TR, Paster BJ, Stokes LN, Susarla SM, Shanti RM. Molecular methods for diagnosis of odontogenic infections. J Oral Maxillofac Surg. 2012;70(8):1854-9.

6. Flynn TR. Antimicrobial treatment of head and neck infections. In: Bagheri SC, Bell RB, Khan HA, editors. Current therapy in oral and maxillofacial surgery. Philadelphia: Elsevier Saunders; 2012. p. 1068-80.

7. Dewhirst FE, Chen T, Izard J, Paster BJ, Tanner AC, Yu WH, et al. The human oral microbiome. J Bacteriol. 2010;192(19):5002-17.

8. Alcaide ML, Bisno AL. Pharyngitis and epiglottitis. Infect Dis Clin N Am. 2007;21(2):449-69, vii.

9. Holm K, Bank S, Nielsen H, Kristensen LH, Prag J, Jensen A. The role of Fusobacterium necrophorum in pharyngotonsillitis - a review. Anaerobe. 2016;42:89-97.

10. Boscolo-Rizzo P, Marchiori C, Montolli F, Vaglia A, Da Mosto MC. Deep neck infections: a constant challenge. ORL J Otorhinolaryngol Relat Spec. 2006;68(5):259-65. 
11. Brook I. Microbiology and management of peritonsillar, retropharyngeal, and parapharyngeal abscesses. J Oral Maxillofac Surg. 2004;62(12):1545-50.

12. Larawin V, Naipao J, Dubey SP. Head and neck space infections. Otolaryngol Head Neck Surg. 2006;135(6):889-93.

13. Klug TE. Incidence and microbiology of peritonsillar abscess: the influence of season, age, and gender. Eur J Clin Microbiol Infect Dis. 2014;33(7):1163-7.

14. Harris AM, Hicks LA, Qaseem A. Appropriate antibiotic use for acute respiratory tract infection in adults: advice for high-value care from the American College of Physicians and the Centers for Disease Control and Prevention. Ann Intern Med. 2016;164(6):425-34.

15. Shulman ST, Bisno AL, Clegg HW, Gerber MA, Kaplan EL, Lee G, et al. Clinical practice guideline for the diagnosis and management of group A streptococcal pharyngitis: 2012 update by the Infectious Diseases Society of America. Clin Infect Dis. 2012;55(10):1279-82.

16. Spinks A, Glasziou PP, Del Mar CB. Antibiotics for sore throat. Cochrane Database Syst Rev. 2013;(11):Cd000023.

17. Baugh RF, Archer SM, Mitchell RB, Rosenfeld RM, Amin R, Burns JJ, et al. Clinical practice guideline: tonsillectomy in children. Otolaryngol Head Neck Surg. 2011;144(1 Suppl):S1-30.

18. Morad A, Sathe NA, Francis DO, McPheeters ML, Chinnadurai S. Tonsillectomy versus watchful waiting for recurrent throat infection: a systematic review. Pediatrics. 2017;139(2):e20163490.

19. Cabrera CE, Deutsch ES, Eppes S, Lawless S, Cook S, O'Reilly RC, et al. Increased incidence of head and neck abscesses in children. Otolaryngol Head Neck Surg. 2007;136(2):176-81.

20. Cheng J, Elden L. Children with deep space neck infections: our experience with 178 children. Otolaryngol Head Neck Surg. 2013;148(6):1037-42.

21. Plum AW, Mortelliti AJ, Walsh RE. Microbial Flora and Antibiotic resistance in Peritonsillar abscesses in upstate New York. Ann Otol Rhinol Laryngol. 2015;124(11):875-80.

22. Page C, Biet A, Zaatar R, Strunski V. Parapharyngeal abscess: diagnosis and treatment. Eur Arch Otorhinolaryngol. 2008;265(6):681-6.

23. Souza DL, Cabrera D, Gilani WI, Campbell RL, Carlson ML, Lohse CM, et al. Comparison of medical versus surgical management of peritonsillar abscess: a retrospective observational study. Laryngoscope. 2016;126(7):1529-34.

24. Wong DK, Brown C, Mills N, Spielmann P, Neeff M. To drain or not to drain - management of pediatric deep neck abscesses: a casecontrol study. Int J Pediatr Otorhinolaryngol. 2012;76(12):1810-3.
25. Saluja S, Brietzke SE, Egan KK, Klavon S, Robson CD, Waltzman ML, et al. A prospective study of 113 deep neck infections managed using a clinical practice guideline. Laryngoscope. 2013;123(12): 3211-8.

26. Novis SJ, Pritchett CV, Thorne MC, Sun GH. Pediatric deep space neck infections in U.S. children, 2000-2009. Int J Pediatr Otorhinolaryngol. 2014;78(5):832-6.

27. Anaya DA, Dellinger EP. Necrotizing soft-tissue infection: diagnosis and management. Clin Infect Dis. 2007;44(5):705-10.

28. Wall DB, de Virgilio C, Black S, Klein SR. Objective criteria may assist in distinguishing necrotizing fasciitis from nonnecrotizing soft tissue infection. Am J Surg. 2000;179(1):17-21.

29. Thomas AJ, Meyer TK. Retrospective evaluation of laboratory-based diagnostic tools for cervical necrotizing fasciitis. Laryngoscope. 2012;122(12):2683-7.

30. Wall DB, Klein SR, Black S, de Virgilio C. A simple model to help distinguish necrotizing fasciitis from nonnecrotizing soft tissue infection. J Am Coll Surg. 2000;191(3):227-31.

31. Shaikh N, Ummunissa F, Hanssen Y, Al Makki H, Shokr HM. Hospital epidemiology of emergent cervical necrotizing fasciitis. J Emerg Trauma Shock. 2010;3(2):123-5.

32. Beldman TF, Teunisse HA, Schouten TJ. Septic arthritis of the hip by Fusobacterium necrophorum after tonsillectomy: a form of Lemierre syndrome? Eur J Pediatr. 1997;156(11):856-7.

33. Woods CR, Cash ED, Smith AM, Smith MJ, Myers JA, Espinosa CM, et al. Retropharyngeal and Parapharyngeal abscesses among children and adolescents in the United States: epidemiology and management trends, 2003-2012. J Pediatric Infect Dis Soc. 2016;5(3):259-68.

34. Wilson CD, Kennedy K, Wood JW, Kumar TK, Stocks RM, Thompson $\mathrm{RE}$, et al. Retrospective review of management and outcomes of pediatric descending mediastinitis. Otolaryngol Head Neck Surg. 2016;155(1):155-9.

35. Shah RK, Chun R, Choi SS. Mediastinitis in infants from deep neck space infections. Otolaryngol Head Neck Surg. 2009;140(6):936-8.

36. Gewitz MH, Baltimore RS, Tani LY, Sable CA, Shulman ST, Carapetis J, et al. Revision of the Jones Criteria for the diagnosis of acute rheumatic fever in the era of Doppler echocardiography: a scientific statement from the American Heart Association. Circulation. 2015;131(20):1806-18.

37. Carapetis JR, Steer AC, Mulholland EK, Weber M. The global burden of group A streptococcal diseases. Lancet Infect Dis. 2005;5(11):685-94. 